\title{
Modeling of high-current source-gated transistors in amorphous silicon
}

\author{
F. Balon, ${ }^{\text {a) }}$ J. M. Shannon, and B. J. Sealy \\ Advanced Technology Institute, SEPS, University of Surrey, Guildford, GU2 7XH, United Kingdom
}

(Received 20 July 2004; accepted 20 December 2004; published online 8 February 2005)

\begin{abstract}
Compared with the field-effect transistor, the source-gated transistor has a much lower saturation voltage and higher output impedance. These features are investigated using computer modeling for amorphous silicon transistors operated at high currents when source barriers are low. In particular, it is shown that low saturation voltages are maintained at high current and are insensitive to source-drain separation. Furthermore, the output impedance is preserved even for submicron source-drain separations. (C) 2005 American Institute of Physics. [DOI: 10.1063/1.1865348]
\end{abstract}

The source-gated transistor (SGT) is a unipolar device that has a much lower saturation voltage and higher output impedance than a field-effect transistor (FET). ${ }^{1,2}$ It is easy to make using thin film technology since one layer can be grown on another in any order. Here we are concerned with the SGT transistor in hydrogenated amorphous silicon $(a-\mathrm{Si}: \mathrm{H})$, the most commonly used semiconductor for large area electronics. It has been shown that saturation voltage can be as much as a factor of 10 lower in an amorphous silicon SGT compared with a FET ${ }^{2}$ which has important implications for device circuits such as those used to address displays based on organic light emitting diodes. The low saturation voltage enables transistors to be operated at lower drain voltages and with a correspondingly lower power dissipation. Furthermore, the SGT in $a-\mathrm{Si}: \mathrm{H}$ is more stable to voltage-temperature stressing than an equivalent FET. ${ }^{3}$ Here we are concerned with modeling of the SGT to establish whether the low saturation voltage and high output impedance are preserved at the currents needed to drive light emitting diode arrays. These currents are typically in the microamp region.

A schematic cross section on a SGT is shown in Fig. 1. The principle of the operation of the SGT differs from that of the FET where the gate voltage modulates channel conductance and saturation occurs when the drain region is depleted. In the SGT the current is determined by a reverse biased barrier and the effect of the electric field. As the drain voltage increases the depletion region spreads until it reaches the semiconductor-insulator $(S-I)$ interface. At this drain bias point $\left(V_{\mathrm{SAT}}\right)$ the current saturates because further increase in drain voltage only extends the depletion layer towards the drain contact and there is little effect on the electric field at the barrier. The values of $V_{\mathrm{SAT}}$ can be small if the semiconductor is thin and lightly doped. In the saturated condition the electric field and the current passing through the source are controlled by the gate. Therefore the on current is controlled by the effect of the gate voltage on the reverse biased source barrier of length $s$. The off current, however, is determined by the parasitic FET of length $d$ in series with the gated source (Fig. 1). A chromium Schottky barrier modified using ion implantation (phosphorous $10 \mathrm{keV}, 1 \times 10^{14} \mathrm{~cm}^{-2}$ ) was used for the source barrier and a compensating implant $\mathrm{BF}_{2}\left(13 \mathrm{keV}, 5 \times 10^{13} \mathrm{~cm}^{-2}\right)$ was used to passivate and pro-

\footnotetext{
${ }^{a}$ Author to whom correspondence should be addressed; electronic mail:
} f.balon@surrey.ac.uk vide field relief at the edge of the source barrier. ${ }^{2}$ In this device the electric field at the source barrier determines the magnitude of the electron current passing through the barrier due to thermionic-field emission.

The experimental transistor characteristics of a SGT are shown in Fig. 2. The thickness of $a-\mathrm{S}: \mathrm{H}$ and silicon nitride was 100 and $300 \mathrm{~nm}$, respectively. It can be seen that the SGT has a low saturation voltage $\left(V_{\mathrm{SAT}}\right)$ and high output impedance. Assuming uniform emission over the area of the source barrier, the current density and the effective barrier height, $\Phi_{B}$ of the source was determined from the measured transistor characteristics. According to the model for the SGT based on thermionic-field emission, ${ }^{2}$ the transconductance $g_{m}$ should be proportional to the source current $I_{s}$

$$
g_{m}=I_{S} \alpha q C_{S} C_{G} / \varepsilon_{S} \varepsilon_{0} k T\left(C_{S}+C_{G}\right),
$$

where $I_{s}$ is the current through the reverse biased barrier, $C_{s}$ and $C_{G}$ are capacitances per unit area of the semiconductor and the insulator, respectively, and $\alpha$ is a tunneling constant.

Based on Eq. (1) the tunneling constant $\alpha$ was found to be $\sim 3 \mathrm{~nm}$ which is in good agreement with values obtained using various amorphous silicon Shottky diodes. ${ }^{4}$ Furthermore, since

$$
I_{S}=S A^{*} T^{2} \exp \left(-q / k T\left[\Phi_{B}-\alpha\left(V_{\mathrm{SAT}} / t_{a-\mathrm{Si}}\right)\right]\right.
$$

and

$$
V_{\mathrm{SAT}}=C_{G}\left(V_{G}-V_{T}\right) /\left(C_{G}+C_{S}\right)+K
$$

the effective barrier height $\Phi_{B}$ can be easily estimated. $S$ and $t_{a-\mathrm{Si}}$ are the area of the source and thickness of the semiconductor (Fig. 1). $V_{T}$ is the threshold voltage of the parasitic FET and $K$ is a constant, which takes into account the drain voltage required to deplete the semiconductor from charge at the $S$-I interface.

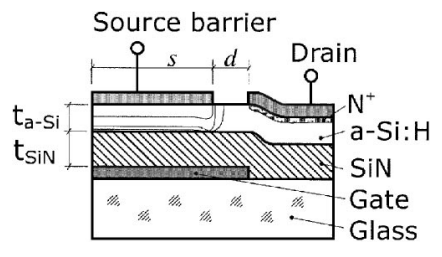

FIG. 1. Schematic drawing of a SGT structure used in simulations showing the spreading of the reverse biased source barrier depletion region towards the semiconductor-insulator interface. 


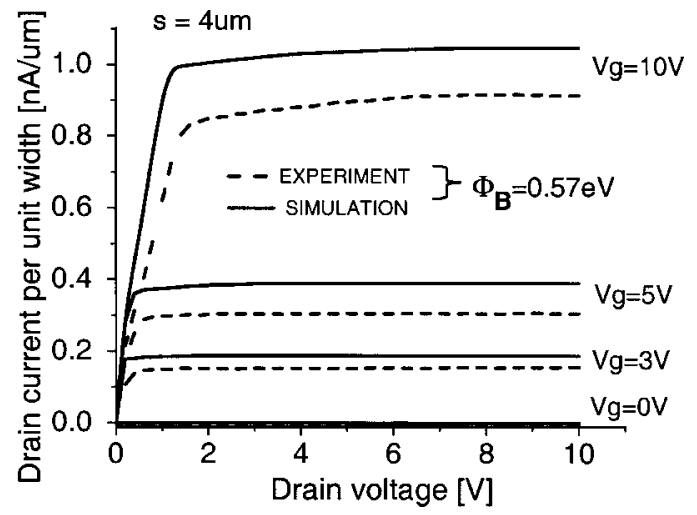

FIG. 2. Normalized transistor characteristic per unit width of experimental and simulated SGT devices.

The barrier height of $0.57 \mathrm{eV}$ determined from these experimental characteristics was used as an input data for the simulations. All simulations were done with the developer version of the ATLAS Silvaco (v.5.7.28.C). The magnitude and shape of the characteristics for simulated and experimental transistor characteristics per unit width are found to be in very good agreement (Fig. 2) particularly in view of the sensitivity of the current to the barrier height.

While agreement between the magnitude of the current obtained from modeling was in good agreement with measured values for high source barriers and low currents, this was not the case when the source barrier was low and currents much higher. It was found that modeling always produced higher currents. This discrepancy is probably due to a change in the field dependence of the current for high fields and low barriers and the fact that emission is no longer uniform over the source area but confined to the edge of the source. For high current devices, simulations showed a lateral voltage drop along the $S$ - $I$ interface responsible for current crowding near the source edge opposite the drain contact. However, the shape of the transistor characteristics at higher currents remained in good agreement with that modeled particularly around saturation (see Fig. 3).

The transistor characteristics for a high current device $\left(2.5 \times 10^{-7} \mathrm{~A} / \mu \mathrm{m}\right)$ are shown in Fig. 3. We see that the low saturation voltage is preserved albeit less well defined in comparison with low current device (see Fig. 2). Furthermore, the output impedance remains high with very little deterioration even for submicron source-drain separations.

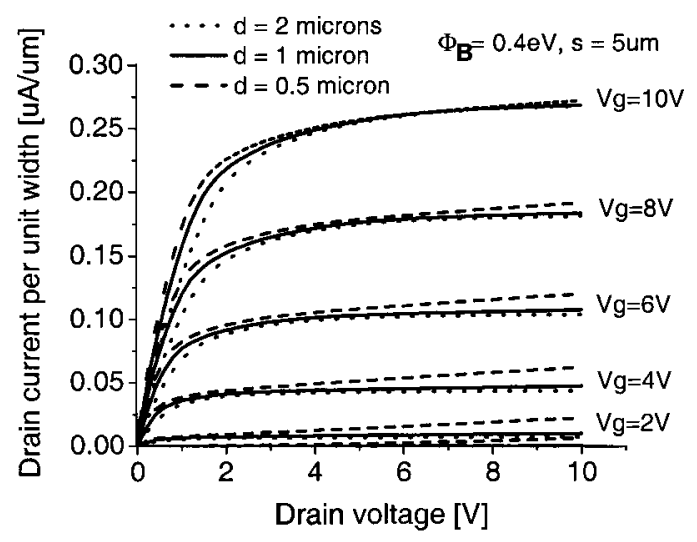

FIG. 3. Simulated SGT transistor characteristics for high current devices

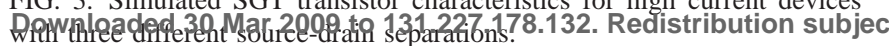

This feature illustrates the good screening of the source from the drain field provided by this geometry.

The good modeling results around saturation enabled us to predict the effect of the source-drain separation and the parasitic FET in series with the source (channel length $d$ in Fig. 1). Clearly for a given source current the characteristics will become increasingly influenced by the parasitic FET as $d$ increases and the current becomes limited by the FET. This feature is shown experimentally in Fig. 4(a) for a high barrier device $\left(t_{a-\mathrm{Si}}=100 \mathrm{~nm}\right)$. For low source-drain separations the change of $V_{\mathrm{SAT}}$ with $V_{G}$ is in good agreement with the dielectric model for the $\mathrm{SGT}^{2}\left[d V_{\mathrm{SAT}} / d V_{G}=C_{G} /\left(C_{G}+C_{S}\right)\right]$ while for large separations it tends to the FET limit for long channel devices $\left(d V_{\mathrm{SAT}} / d V_{G}=1\right){ }^{5}$ Computer calculations [Fig. 4(b)] show the same behavior $\left(t_{a-\mathrm{Si}}=60 \mathrm{~nm}\right)$. Overall these results show that $V_{\mathrm{SAT}}$ is not strongly dependent on $d$ and wide variations can be tolerated without large changes in $V_{\text {SAT }}$. For example, for a source-drain separation as large as $10 \mu \mathrm{m} d V_{\mathrm{SAT}} / d V_{G}$ is still four times lower than a FET.

In conclusion, it has been shown that the shape of the output characteristics of a SGT having a Shottky barrier source can be predicted using the SILVACO Atlas program. It is found that a low $V_{\mathrm{SAT}}$ is maintained when the source barriers are low and the current is high. The saturation voltage is less well defined but the low values should enable circuits to be operated at lower drain voltages and power dissipation. The saturation voltage increases with source drain separation because the current becomes restricted by the parasitic FET in the extrinsic drain region. However, this effect is less pronounced than expected. It has also been shown that SGT characteristics can be preserved down to submicron sourcedrain separation.
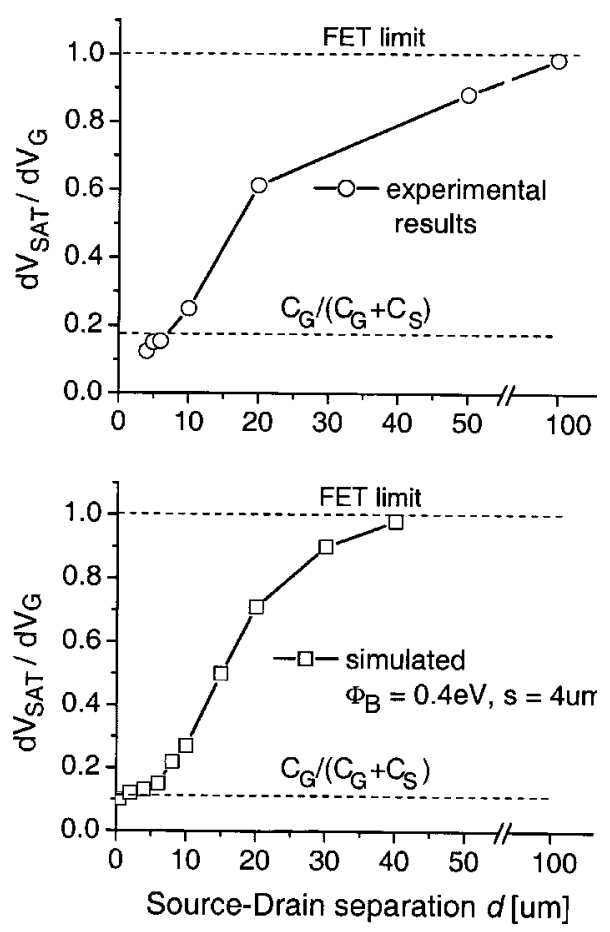

FIG. 4. The change in saturation voltage with gate voltage for SGTs with a range of source-drain separation (a) experimental and (b) simulated results

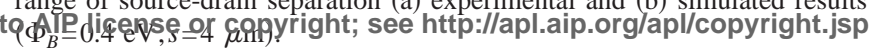


The $a$-Si:H transistors used in this work were made at Philips Research Laboratories, Redhill, Surrey, UK. The project is supported by EPSRC, Swindon, UK.

${ }^{1}$ J. M. Shannon and E. G. Gerstner, IEEE Electron Device Lett. 24, 405 (2003).
${ }^{2}$ J. M. Shannon and E. G. Gerstner, Solid-State Electron. 48, 1155 (2004). ${ }^{3}$ J. M. Shannon, Appl. Phys. Lett. 85, 326 (2004).

${ }^{4}$ K. J. B. M. Nieuwesteeg, M. van der Veen, T. J. Vink, and J. M. Shannon, J. Appl. Phys. 74, 2581 (1993).

${ }^{5}$ S. M. Sze, Physics of Semiconductor Devices, 2nd ed. (Wiley Interscience, New York, 1981), Chap. 6. 